\title{
Toward Better Light-Confinement in Micropillar Cavities
}

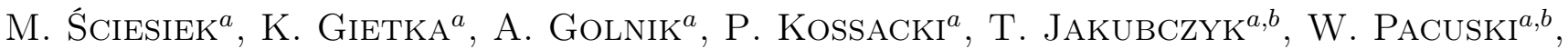 \\ C. KRUSE ${ }^{b}$ AND D. HOMMEL ${ }^{b}$ \\ ${ }^{a}$ Institute of Experimental Physics, Faculty of Physics, University of Warsaw, Hoża 69, 00-681 Warsaw, Poland \\ ${ }^{b}$ Institute of Solid State Physics, University of Bremen, Postfach 330 440, D-28334 Bremen, Germany
}

We report on a two-step etching of ZnTe based micropillars. We demonstrate applicability of the technology and we analyze the optical properties of obtained structures. Microphotoluminescence spectra of individual micropillars show a typical mode pattern that confirms a successful growth of photonic structures. The reflectivity and photoluminescence spectra of a planar microcavity measured for various incident angles show that additional side distributed Bragg reflectors will be important for the further enhancement of photon confinement in micropillar cavity.

PACS: 78.67.Pt, 42.70.Qs, 42.79.Fm, 78.66.Hf

\section{Introduction}

Semiconductor quantum dots (QDs) embedded in photonic structures called micropillars have been studied for several years [1]. The interest is caused by such properties as high photon extraction efficiency or the Purcell effect [2]. A usual micropillar consists of a microcavity embedded between two distributed Bragg reflectors (DBRs) on the top and on the bottom. The planar confinement is caused by etching the pillars out of the sample. In such a case planar confinement is caused by the reflection on the border of high refractive micropillar's material and the low refractive index of the air or vacuum. However, there is still some leaking of photons horizontally [3]. This leakage might be suppressed by surrounding the micropillar horizontally by another DBR. In this paper we present steps toward obtaining micropillars with lateral DBRs which in the future should lead to a better confinement of light inside the microcavity. Thanks to the expected low volume of such a microcavity and the high quality factor $Q$ one can significantly enhance light-matter coupling.

\section{Sample preparation}

The first step in the sample preparation was a MBE growth of the ZnTe based planar microcavity with the CdTe quantum dots $[4,5]$. Growth was carried out on GaAs substrate. In the Bragg reflectors the $\mathrm{ZnTe}$ was the layer with high refractive index and the short-period superlattice $\mathrm{ZnTe} / \mathrm{MgSe} / \mathrm{ZnTe} / \mathrm{MgTe}$ was the layer with low refractive index [6]. The planar DBRs fabricated for our micropillars have a wide stop-band within the range of QDs photoluminescence. A very thin band pass (cavity mode) is formed in the middle of the stop-band. By embedding the QDs into the cavity one expects an enhancement of microluminescence for this resonant energy. The second step of the technology was a deposition of a photoresist and an optical exposition through a mask.
Later, the sample was exposed to the ion etching resulting in a fast emergence of a large number of micropillars of $10 \mu \mathrm{m}$ diameter. The next step was the focused ion beam (FIB) etching which reduced the size of the individual micropillar to $1-5 \mu \mathrm{m}$ in the diameter. The combination of both etching techniques allows an efficient creation of a large number of micropillars standing on the samples' surface. The use of precise but expensive and time-consuming FIB etching is reduced to a minimum. As a technique for this ongoing step laser pulsed deposition is proposed as it enables uniform coating of sides of such free standing columns [7]. An example of a sample prepared with our mixed method is presented in Fig. 1.

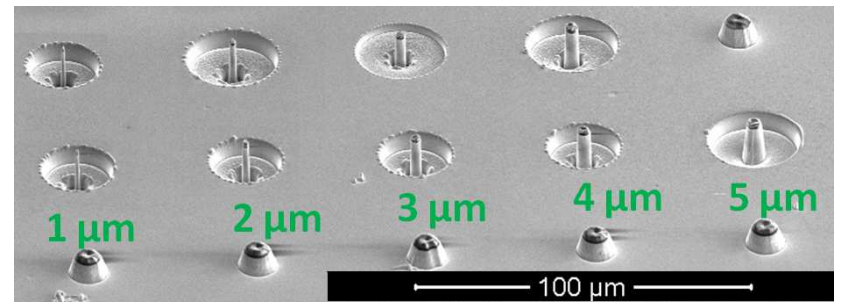

Fig. 1. Scanning electron microscopy (SEM) image of the sample prepared with ion etching and subsequently FIB. First the $10 \mu \mathrm{m}$ micropillars are prepared with ion etching (as shown in the lowest row). Later some of them are individually etched using FIB (as shown in the middle row). The labels below micropillars correspond to their diameter.

\section{Experimental setup}

For the microphotoluminescence measurements we placed the samples in a helium flow cryostat, above which an objective lens on a $x-y-z$ piezoelectric positioner was mounted. The piezoelectric positioner allowed very precise movement over micropillars. Micropillars were ex- 
cited by a green or a blue laser. The emitted microphotoluminescence was analyzed with a spectrometer equipped with a CCD camera. The FIB etching of special marks on the sample allowed identification of individual micropillars using the optical imaging technique. The optical imaging was executed with the use of another CCD camera which recorded the image of the sample illuminated by a halogen lamp. This allowed localization of micropillars and an adjustment of their position. When this step was done the lamp was turned off, the laser was turned on, and the final adjustments for the improvement of the PL signal were made using piezoelectric positioner. A laser cutoff filter was placed in front of the monochromator and a set of lenses allowed the adjustment of the desired collimation of the beams and the focusing of the signal on the monochromator slit.

\section{Results}

In order to estimate the impact of the photonic environment on the photoluminescence, the spectra related to the planar microcavity were measured on unprocessed part of the sample. The data from measuring two different angles (perpendicular and parallel to the samples surface) was collected. Additionally, the reflectivity perpendicular to the sample's surface was measured. The typical results are presented in Fig. 2.
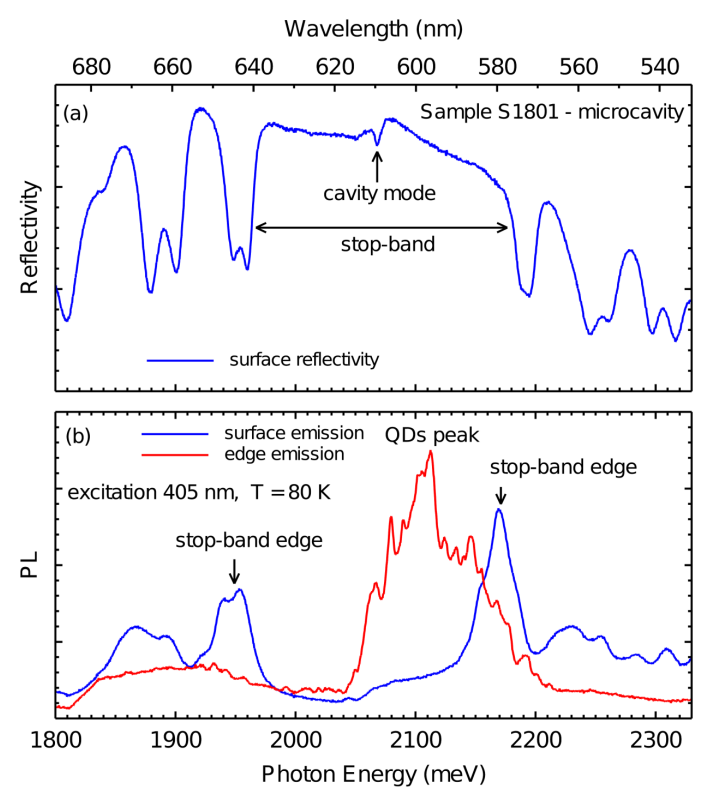

Fig. 2. Optical properties of planar microcavity: (a) Reflectivity spectrum measured from the surface of the sample; (b) the photoluminescence spectra measured from the surface and from the edge of the sample.

Figure 2a presents the stop-band and the cavity mode in reflectivity. Figure $2 \mathrm{~b}$ presents the corresponding stop-band edges and the QDs peak in photoluminescence. When observing surface emission one can see that its pattern is similar to a constant with subtracted reflectivity

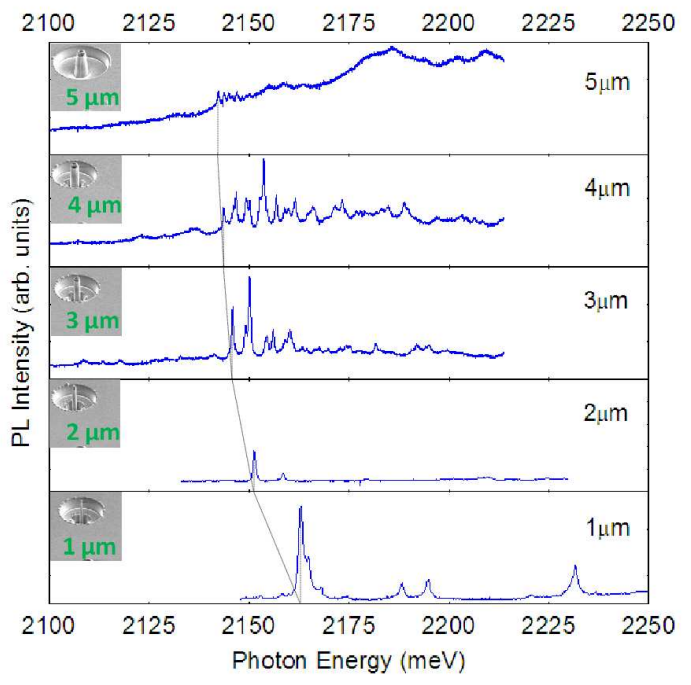

Fig. 3. Photoluminescence spectra of micropillars with various diameters. Excitation at $532 \mathrm{~nm}, T=60 \mathrm{~K}$. With decreasing diameter the shifting of fundamental mode toward higher energies is observed as expected. The magnitude of modes' width at half the maximum in the range of a few tenths of a meV shows very high quality $(Q \approx 4000)$ of produced micropillars.

curve. This corresponds to the transmission coefficient of the light coming out of the sample. We conclude that this is because the QDs are excited but cannot emit light efficiently in energies which are highly reflected. Such behavior is expected for QDs in planar microcavity. The edge emission has a characteristic radiation pattern of the QDs' PL unchanged by the photonic environment. This shows that QDs tuned to the cavity mode might still emit horizontally. The additional lateral confinement would enhance both the photon confinement in micropillars and the light-matter coupling. In order to verify the quality of etching the photoluminescence of single micropillars was measured. Observed mode structure (Fig. 3) confirms that using two-step etching results in a very good quality of micropillars ( $Q$ factor in the range of 4000). We can also see that with the decreasing diameter of a pillar the fundamental mode shifts toward higher energies, as expected for such structures [2].

\section{Conclusions}

The technique of ion etching allows fast preparation of a large number of micropillars in one process. They might be later treated carefully with FIB. Therefore, two step etching results in high quality micropillars surrounded by a large empty area. Such area is necessary for placing of the lateral DBRs which are essential to improve light-confinement in micropillars since edge emission is not affected by the photonic environment. Efficient decrease of the coupling of the emission to the non-resonant energies (leaky modes) is expected. 


\section{Acknowledgments}

This work was supported by Polish public funds in years 2010-2014: project Iuventus Plus of the Ministry of Science and Higher Education and project Lider of the National Centre for Research and Development $(\mathrm{NCBiR})$.

\section{References}

[1] A.J. Shields, Nature Photon. 1, 215 (2007).

[2] J.M. Gérard, D. Barrier, J.Y. Marzin, R. Kuszelewicz, L. Manin, E. Costard, V. Thierry-Mieg, T. Rivera, Appl. Phys. Lett. 69, 449 (1996).

[3] J.M. Gérard, B. Sermage, B. Gayral, B. Legrand, E. Costard, V. Thierry-Mieg, Phys. Rev. Lett. 81, 1110 (1998).
[4] C. Kruse, W. Pacuski, T. Jakubczyk, J. Kobak, J.A. Gaj, K. Frank, M. Schowalter, A. Rosenauer, M. Florian, F. Jahnke, D. Hommel, Nanotechnology 22, 285204 (2011).

[5] J. Kobak, W. Pacuski, T. Jakubczyk, T. Kazimierczuk, A. Golnik, K. Frank, A. Rosenauer, C. Kruse, D. Hommel, J.A. Gaj, Acta Phys. Pol. A 119, 627 (2011).

[6] W. Pacuski, C. Kruse, S. Figge, D. Hommel, Appl. Phys. Lett. 94, 191108 (2009).

[7] H. Hilmer, J. Sellmann, Ch. Sturm, R. Schmidt-Grund, B. Rheinländer, H. Hochmuth, J. Lenzner, M. Lorenz, M. Grundmann, AIP Conf. Proc. 1199, $151(2010)$. 